\title{
Non Rheumatic Atrial Fibrillation as Risk of Stroke
}

\author{
Ali Areef Fadhlullah ${ }^{1,2}$, Asgad A. Abdalgbar ${ }^{1,2}$, Hanan K. Altalhi ${ }^{1,2}$ \\ ${ }^{1}$ Faculty of Medicine, University of Omar El mukhtar, Albayda, Libya \\ ${ }^{2}$ Department of Internal Medicine, University of Omar El mukhtar, Albayda, Libya
}

Email address:

ali.aref67@yahoo.com (A. A. Fadhlullah), aabdalgbar@yahoo.com (A. A. Abdalgbar), hnangk@yahoo.com (H. K. Altalhi)

To cite this article:

Ali Areef Fadhlullah, Asgad A. Abdalgbar, Hanan K. Altalhi. Non Rheumatic Atrial Fibrillation as Risk of Stroke. American Journal of Internal Medicine. Vol. 4, No. 6, 2016, pp. 117-119. doi: 10.11648/j.ajim.20160406.15

Received: October 21, 2016; Accepted: November 1, 2016; Published: November 25, 2016

\begin{abstract}
Atrial fibrillation (AF) is one of the main risk factor for ischemic stroke. The reason for an increased stroke risk in $\mathrm{AF}$ has always been claimed to be the occurrence of left atrial thrombosis causing arterial embolism. In patients with Rheumatic heart disease especially mitral valve stenosis with AF, the frequency of atrial thrombosis has found to be $30-42 \%$ (Keren $\mathrm{G}$ et al. 1987), and the prevalence of left atrial thrombosis in NRAF are higher than in control 13-27\% (Petersen P et al. 1988). Objectives: We investigated if there are any differences in risk factors or atherosclerotic manifestations between ischemic brain infarction patients with and without AF? Are the brain lesions characteristics in these patients similar?Patients and Methods: This is a case- control study of 26 patients with acute ischemic stroke and NRAF (case subjects) a and 26 patients with acute ischemic stroke and sinus rhythm. (control subjects). The patients admitted to the hospital; the diagnosis of cerebral infarction was confirmed by new $\mathrm{CT}$ of the brain. All the participants underwent the standard examination and testing as well as ECG and ECHO. Result: Patient with NRAF had higher mortality $30 \%$ than in control (SR) 7\% (P<0.001). NRAF patients had positive brain CT finding $68 \%$ compared to $23 \%$ of the SR Patients $(\mathrm{P}<0.001)$. Conclusion: Brain infarction in non-Rheumatic $\mathrm{AF}$ group are more serious than other and therefor make up a (high risk) group for which acute treatment would be specially indicated and primary prophylaxis with anticoagulants might therefore be considered.
\end{abstract}

Keywords: Cerebral Vascular Accident (CVA), Non Rheumatic Atrial Fibrillation (NRAF), Sinus Rhythm (SR), Electrocardiography (ECG), Echocardiography (ECHO), Atrial Fibrillation (AF)

\section{Introduction}

Atrial fibrillation has been shown to be associated with increased risk for cardiovascular disease morbidity (Britton M, et al. 1985) and mortality (Kannel WB, Et al. 1982). Although the importance of AF partly derives from its strong association with ischemic stroke. There have not been as many advances in our understanding of the mechanism of stroke in AF. Current views rest on a century old hypothesis that fibrillation of the atrium produce stasis of blood which cause thrombosis formation and embolism to the brain. When other abnormalities are acknowledged to play role, the dysrhythmia is still considered the primary cause of, thromboembolism (Zimetbaum P, et al. 2014). Non rheumatic atrial fibrillation carries a nearly fivefold increased risk for stroke compared with normal sinus rhythm. (Wolf PA, et al. 1991).

\section{Objectives}

We investigated if there are any differences in Risk factors or atherosclerotic manifestations between brain infarction patients with and without AF?

Are the brain lesions characteristics in these patients similar?

\section{Patients and Methods}

This is case - control study of patients admitted to the hospital with diagnosis of acute ischemic infarction. Data were collected with interview the cases, physical and neurological examination done by study physicians. The diagnosis of ischemic stroke was confirmed by new computer Tomography (CT).

ECG done for every CVA patients as interpreted by 
cardiologist from standard 12 leads ECG, the patients were divided to two groups according to the ECG finding, chronic AF ( $\mathrm{n}=26)$, constant AF during hospital stay, sinus rhythm ( $\mathrm{n}=26)$-not known episodes of AF during hospital stay.

The ECG was appraised according to the Minnesota code (The Scandinavian committee on ECG classification).

Echo: Transthoracic echocardiography (TTE) was performed in all subjects and assessed by cardiologist. 2D, M - Mode - PW - CW were performed to asses LVEF, was measured by simplified cylinder - hemielliposid formula (Weyman AE, Et al. 1994) and excluded the Rheumatic heart Valve disease.

\section{Other various Risk factors:}

BP measurement in right arm, Cigarette smoking to be qualified based on daily consumption and duration of smoking, blood biochemistry analysis were made ie. Fasting blood sugar (FBS) total cholesterol, TG, CBC.

Statistical analysis:

Statistical analysis were performed by using SPSS software (version 11) difference among groups were analyzed by one way. unstack ANOVA and $\mathrm{P}$ values 0.05 were considered significant.

\section{Result}

Table 1: shows Characteristics and variation of study subjects: 52 patients with acute ischemic stroke were included in the study, 26 patient with NRAF (case subject ) and 26 patient with SR (control subjects). There is significant difference in mean age between NRAF group and SR group

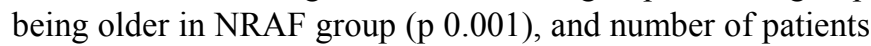
with history of diabetes and heart failure is significantly increased among NRAF group ( $\mathrm{p}$ 0,007, p 0.001 respectively).

Table 1. Study population characteristics and variation.

\begin{tabular}{ll|ll}
\hline & AF & SR & P Value \\
\hline Number & 26 & 26 & \\
\hline Age mean \pm SD & $68.96 \pm 15.46$ & $52.96 \pm 16.39$ & 0.001 \\
Rang & $40-90$ & $30-75$ & \\
Male & $14(53.8 \%)$ & $17(65.4 \%)$ & $0.406(\mathrm{NA})$ \\
Female & $12(46.2 \%)$ & $9(34.6 \%)$ & $0.406(\mathrm{NA})$ \\
Diabetic & $16(61.5 \%)$ & $7(27 \%)$ & 0.007 \\
Hypertension & $8(30.8 \%)$ & $4(15.4 \%)$ & $0.162(\mathrm{NA})$ \\
\hline Smoking & $9(34.6 \%)$ & $11(42.3 \%)$ & $0.724(\mathrm{NA})$ \\
\hline
\end{tabular}

Table 2: delineates the clinical, Laboratory, ECHO and ECG data of study groups: left ventricular dysfunction of any degree was more frequent in $\mathrm{AF}$ patients $(25 \%)$ than in SR patients $(7.5 \%)$. ( $\mathrm{P}=-0.0001)$, other risk factors, measured mean SBP as well as DBP level, FBS, total serum cholesterol, triglyceride, showed no significant difference between groups.
Table 2. Clinical, Laboratory, ECHO and ECG data of study groups.

\begin{tabular}{llll}
\hline & AF $\mathbf{n}=\mathbf{2 6}$ & SR $\mathbf{n}=\mathbf{2 6}$ & P value \\
\hline Initial SBP mmHg & $149.15 \pm 36.65$ & $148.08 \pm 37.31$ & NA \\
Initial DBP mmHg & $92.69 \pm 25.07$ & $88.27 \pm 16.31$ & NA \\
ECG \% & & & \\
Normal (except rhythm) & 7 & 16 & P $<0.05$ \\
Sign MI & 8 & 5 & NA \\
LVH & 3 & 4 & NA \\
LBBB & 5 & 2 & NA \\
Echo EF \% & & & \\
Normal & $16(61 \%)$ & $23(88 \%)$ & NA \\
LV dysfunction & $10(25 \%)$ & $3(7.5 \%)$ & P 0.0001 \\
FBS & $150 \pm 61.69$ & $127.20 \pm 76.98$ & NA \\
Cholesterol & $91.33 \pm 25.88$ & $102.14 \pm 42.12$ & NA \\
Triglycerides & $91.33 \pm 25.88$ & $102.14 \pm 42.12$ & NA \\
\hline
\end{tabular}

Myocardial infarction (MI), Left ventricular hyperatrophy (LVH), Left ventricle (LV)

Table 3: Shows Brain lesions characteristics. And condition on admission: In brain. infarction patients with and without $\mathrm{AF}$ the symptoms were known to have occurred suddenly in $60 \%$ of cases in groups. Paresis was the dominating symptoms. Seizures in the first hour occurred in (11\%) of the AF group compared to (4\%) of SR group $(\mathrm{P}>0.05)$, impaired level of consciousness occurred in $(31 \%)$ of the AF group compared to $(4 \%)$ of the SR group $(\mathrm{p}<0.01)$, patients cannot walk independently occurred in $(69 \%)$ AF group compared to $(31 \%)$ of the SR group $(p=0.05)$, at admission to hospital the AF group had several signs of more serious brain damage than the SR group.

Mortality was higher among the AF group (30\% vs. $23 \%$ respectively; P. $<0.001$ ).

No difference was seen between the groups as regards the location of the brain lesion and there were more pathological finding at $\mathrm{CT}$ brain in the $\mathrm{AF}$ group (68\%) compared to $(23 \%)$ of SR group $(\mathrm{P}=0.001)$.

Table 3. Brain Lesion Characteristics in 52 Patients with NRAF and with $S R$.

\begin{tabular}{llll}
\hline Condition on admission & AF n=26 & SR n=26 & P value \\
\hline Seizures in the first hours & $3(11 \%)$ & $1(4 \%)$ & $\mathrm{P}<0.05$ \\
Impaired consciousness & $8(31 \%)$ & $1(4 \%)$ & $\mathrm{P}<0.01$ \\
Cannot walk independently & $18(69 \%)$ & $8(31 \%)$ & $\mathrm{P}<0.05$ \\
Mortality & $8(30 \%)$ & $2(7 \%)$ & $\mathrm{P}<0.001$ \\
Brain CT (Positive) & $18(68 \%)$ & $6(23 \%)$ & $\mathrm{P}<0.001$ \\
\hline
\end{tabular}

\section{Discussion}

The AF patients were older and had more serious brain damage than those with SR. Some factors have to be dealt with before discussing whether these and other difference between the groups favors an Athero-thrombotic or embolic origin of stroke in AF patients. We chose to compare a patients with AF with those with SR. It has been claimed that a sudden onset of maximum symptoms in patients with an embolic sources, like AF is typical embolism (Easton JD, et al. 1980; Dalal PM, et al. 1965).

We found progression of symptoms after arrival at hospital to be as common among NRAF patients as among others (roden A et al. 1982). 
AF has been consistently associated with stroke in different cohorts (Wolf PA et al. 1978).

AF frequently co-exists with atrial abnormalities, such as endothelial dysfunction (Cai H, et al. 2002), impaired myocyte function (Frustaci A, ey al. 1997), and mechanical dysfunction of left atrial appendage (Mihm MJ, et al. 2001), these abnormality have been documented in both experimental animal models and in human (Warraich HJ, et al. 2014). could these abnormality also arise independently of $\mathrm{AF}$ and cause stroke. Once AF develops the dysrhythmia cause contractile dysfunction and stasis, which further increase the risk of thromboembolism. In addition over time the dysrhythmia cause structural remodeling of the atrium there by worsening atrial cardiopathy and the risk of thromboembolic. 0there is strong association between AF and embolic stroke. $10 \%$ of patients with lacunar infarction have AF (lodder J, et al. 1990).

The AF patients were moreover older, reflecting the increasing prevalence of AF with increasing age. Arteriosclerotic macro-and microangiopathy progressing with age, might instead be the cause of the stroke as well as the heart disease.

The finding in this study of more severe brain damage in AF patients may also argue in favors of embolism. A sudden interruption of blood flow caused by an embolus might give rise to large infarction than gradual occlusion with opportunity for development of collateral circulation. More sever lesions in embolic stroke have also been found at autopsy (Jorgensen L, et al. 1969).

AF was noted to be associated with worse prognosis (Britton M, et al. 1985). There might be other explanation than embolism for the poor outcome of stroke in AF patients. A decreased cardiac output due to the higher incidence of heart failure might be contributed to a reduced cerebral blood flow when auto regulatory mechanism are impaired as in an ischemic region (Keller TS, et al. 1982).

\section{Conclusion}

We would like to conclude that embolism is a plausible cause of stroke in many NRAF patients. NRAF patients not only have increased incidence of stroke but their stroke are more serious for which acute treatment would be especially indicated. More work is required to determine whether additional marker such as cardiac MRI of tissue fibrosis and computed tomography assessment of left atrial appendage morphology, may better identify the risk of atrial thromboembolism

\section{References}

[1] Britton M, Gustafsson C, Non-rheumatic atrial fibrillation as a risk factor for stroke. $1985 ; 16: 182-188$.

[2] Cai H, Li Z, Goette A, Mera F, Honeycutt C, Feterik K, et al.
Down regulation of endocardial nitric oxide synthase expression and nitric oxide production in atrial fibrillation: potential mechanisms for atrial thrombosis and stroke. Circulation. 2002: 106: 2854-2858.

[3] Easton JD, Sherman DG: Management of cerebral embolism of cardiac origin. Stroke11: 433-442, 1980.

[4] Frustaci A, Chimenti C, Bellocci F. Morgante E, Russo MA, Maseri A. Histological substrate of atrial biopsies in patients with lone atrial fibrillation. Circulation. 1997; 96: 1180-1184.

[5] Heijman J, Voiget N, Nattel S, Dobrev D. Cellular and molecular electrophysiology of atrial fibrillation initiation maintenance and progression Circ Res. 2014; 114: 1483-1499. Doi: 10.1161/ CIRCRESAHA.114.302226.

[6] Kannel WB, Abbott RD, Savage DD, McNamara PM, Epidemiologic features of chronic atrial fibrillation: the Framingham study N Engl J. Med. 1982; 306: 1018-1022.

[7] Kannel WB, Wolf PA, Verter J: manifestations of coronary disease predisposing to stroke: The Framingham Study. JAMA 1983; 250: 2942-2946.

[8] Keren G, Etzion T, Sherez J, Zelcer A, Megidish R, Miller H, Laniado $\mathrm{S}$. Atrial fibrillation and atrial enlargement in patients with mitral stenosis. Am Heart J. 1987; 114: 1146-1155.

[9] Lodder J, Bamford JM, Sandercock PA, Jones LN, Warlow CP. Are hypertension or cardiac embolism likely causes of lacunar infarction? Stroke. 1990; 21: 375-381.

[10] Mihm MJ, Yu F, Carnes CA, Reiser PJ, McCarthy, PM, Van Wagoner DR, et al. Impaired myofrillar energetics and oxidative injury during human atrial fibrillation. Circulation. 2001; 104: 174-180.

[11] Peterson P, Godtfredsen J: Risk factor for stroke in chronic atrial fibrillation. Eur Heart J 1988; 9: 291-294.

[12] Roden A, Britton M: Progression of stroke after arrival at hospital. Acta Neurol Scand 66 (suppl 91): 43, 1982.

[13] The Scandinavian Committee on ECG Classification: The Minnesota code for ECG Classification. Adaption to $\mathrm{Cr}$ leads and modification of the code for ECG recording during and after exercise. Acta Med Scand (suppl): 481, 1967.

[14] Jorgensen L, Torvic A: Ischemic cerebrovascular diseases in an autopsy series. Part 2. Prevalence, location, pathogenesis, and clinical course of cerebral infarcts. J Neurol Sci 9: 285320, 1969.

[15] Warraich HJ, Gandhavadi M, Manning WJ. Mechanical discordance of the left atrium and appendage: a novel mechanism of stroke in paroxysmal atrial fibrillation, Stroke. 2014; 45: 1481-1484, doi: 10.1161/STROKEAHA.114.004800.

[16] Weyman AE. Principles and practice of echocardiography, Lea and Febiger Publ. 194; 606.

[17] Wolf PA, Abbott RD, Kannel WB: Atrial fibrillation: A major contributor to stroke in the elderly. Am Heart J 1986; 112: 1039-1043.

[18] Zimetbaum P, Waks JW, Ellis ER, Glotzer TV, Passman RS. Role of atrial fibrillation burden in assessing thromboembolic risk. Circ. Arrhythm Electrophysiology 2014; 7: 1223-1229. 